\title{
Eurostudia
}

\section{Quand les hommes politiques allemands deviennent objet de fiction : les personnages allemands dans le roman Les aventures extraordinaires de deux Canayens de Jules Jéhin (1918)}

\section{Eve Léger-Bélanger}

Volume 13, numéro 1-2, 2018-2019

Cultures en contact, entre régulations et représentations

URI : https://id.erudit.org/iderudit/1067280ar

DOI : https://doi.org/10.7202/1067280ar

Aller au sommaire du numéro

Éditeur(s)

Le Centre canadien d'études allemandes et européennes

ISSN

1718-8946 (numérique)

Découvrir la revue

Citer cet article

Léger-Bélanger, E. (2018). Quand les hommes politiques allemands deviennent objet de fiction : les personnages allemands dans le roman Les aventures extraordinaires de deux Canayens de Jules Jéhin (1918). Eurostudia, 13(1-2), 193-207. https://doi.org/10.7202/1067280ar
Résumé de l'article

Cet article porte sur les croisements entre fiction et réalité dans le roman Les aventures extraordinaires de deux Canayens (1918) de Jules Jéhin, une oeuvre satirique québécoise qui se déroule pendant la Grande Guerre. Dans le roman, l'Allemagne est un ennemi politique et militaire. Les aventures extraordinaires de deux Canayens fut initialement publié sous la forme de feuilleton dans Le Canard, un journal humoristique et satirique. La représentation fictive de personnages allemands qui ont réellement existé sera tout d'abord examinée. La façon dont le feuilleton entre en résonance avec le reste du journal, enraciné dans la réalité, sera ensuite étudiée. L'objectif est de mettre en relief les différentes manières dont le roman s'ancre dans la réalité de l'époque tout en appartenant à la fiction.
Tous droits réservés ( Le Centre canadien d'études allemandes et européennes, 2019
Ce document est protégé par la loi sur le droit d'auteur. L'utilisation des services d’Érudit (y compris la reproduction) est assujettie à sa politique d'utilisation que vous pouvez consulter en ligne. 
Quand les hommes politiques allemands deviennent objet de fiction: les personnages allemands dans le roman Les aventures extraordinaires de deux Canayens de Jules Jéhin (1918)

\section{Eve Léger-Bélanger}

Université de Montréal

\section{Résumé}

Cet article porte sur les croisements entre fiction et réalité dans le roman Les aventures extraordinaires de deux Canayens (1918) de Jules Jéhin, une œuvre satirique québécoise qui se déroule pendant la Grande Guerre. Dans le roman, l'Allemagne est un ennemi politique et militaire. Les aventures extraordinaires de deux Canayens fut initialement publié sous la forme de feuilleton dans Le Canard, un journal humoristique et satirique. La représentation fictive de personnages allemands qui ont réellement existé sera tout d'abord examinée. La façon dont le feuilleton entre en résonance avec le reste du journal, enraciné dans la réalité, sera ensuite étudiée. L'objectif est de mettre en relief les différentes manières dont le roman s'ancre dans la réalité de l'époque tout en appartenant à la fiction.

\section{Abstract}

This article focuses on the crossroads between fiction and reality in the novel Les Aventures extraordinaires de deux Canayens (1918) by Jules Jéhin, a Quebec satirical work that takes place during the Great War, where Germany is a political and military enemy. Les aventures extraordinaires de deux Canayens was first published as a serial in Le Canard, a 
humorous and satirical newspaper. In this article, the fictional representation of real-life German characters will be first examined. How the fictional serial resonates with the rest of the newspaper itself tied up to reality will then be examined. This article aims to highlight the different ways in which the novel is anchored in reality while belonging to fiction.

La Grande Guerre, qui a marqué la collectivité québécoise, a engendré des représentations de l'Allemagne en tant que figure Autre, mise à distance. Les représentations de cet Autre dans les textes littéraires et de presse québécois à la fin de la guerre sont majoritairement composées d'éléments à caractère essentialisé, comme des stéréotypes, ce qui met en lumière un "processus de négation et d'exclusion » (Lüsebrink 1996 : 65) de l'Allemagne, l'ennemi de guerre.

Un roman québécois publié à la fin de la Grande Guerre est particulièrement intéressant quant à ses représentations de l'Allemagne: Les aventures extraordinaires de deux Canayens ${ }^{1}$ (1918) de Jules Jéhin. Dans cette œuvre satirique dont l'intrigue se déroule pendant la Grande Guerre, l'Allemagne est un ennemi politique et militaire. Grâce à l'humour et à l'ironie, Jéhin déconstruit dans le récit la menace que représente alors l'Allemagne pour les Alliés. Dans le roman, l'Allemagne s'incarne non seulement dans des motifs usuels, comme le service d'espionnage, mais aussi dans des personnages allemands qui ont réellement existé, comme Guillaume II.

Les aventures extraordinaires de deux Canayens paraît initialement sous la forme de roman-feuilleton dans un hebdomadaire montréalais humoristique et

\footnotetext{
${ }^{1}$ L'édition utilisée est la suivante : J. Jéhin, Les aventures extraordinaires de deux Canayens : charivari littéraire et scientifique, Montréal, Imprimerie A-P. Pigeon, 1918, 114 p. Les références à ce roman seront dorénavant notées par le sigle $A E D C$ et le folio, entre parenthèses dans le corps du texte.
} 
satirique appelé Le Canard, dont le tirage est d'environ 7750 exemplaires en 1918 (McKim 1919). Les aventures extraordinaires de deux Canayens est publié dans le journal du 19 mai 1918 au 3 novembre 1918; il se termine une semaine avant l'armistice.

Le roman de Jéhin se déroule en grande partie en 1917, pendant la Grande Guerre. Ainsi, le temps du récit correspond presque exactement au temps réel, sans véritable distance entre les deux. Dans le roman, trois personnages canadiens-français, nommés Baptiste Courtemanche, Titoine Pelquier et Philias Duval, s'associent pour construire en secret un dirigeable révolutionnaire qui défie la gravité et qui peut aller dans l'espace, du jamais-vu, grâce à la découverte d'un minerai (fictif) hors du commun. Les personnages partent alors à l'aventure et créent leur propre État de l'air. Les grandes nations sont alarmées par le dirigeable aux incroyables propriétés dont personne ne connaît l'origine. Le roi de Prusse, Guillaume II, fait alors appel à des agents du renseignement allemand pour tout savoir sur le formidable vaisseau, sans succès. Les personnages canadiens-français, qui veulent rester neutres dans le conflit, utilisent finalement leur technologie afin de détruire un sous-marin allemand qui attaque un navire de la Croix-Rouge. Le roman se termine par le retour des Canadiens français au Québec. Ils révèlent enfin leur identité à tous et sont accueillis en héros.

Dans cette étude, nous proposons d'analyser les croisements entre fiction et réalité dans le roman humoristique québécois de Jéhin. Nous désirons mettre en relief la manière dont l'œuvre est ancrée à la fois dans le réel - d'un point de vue chronologique (1918) et spatial (le journal) - et détachée de celui-ci grâce, entre autres, à l'humour. Nous étudierons les représentations des personnages allemands dans le roman qui ont, pour la plupart, existé. Nous examinerons aussi les caricatures, en nous attachant à ce qu'elles évoquent et au dialogue qu'elles créent entre fiction et réalité dans le journal Le Canard. Tout au long de notre 
réflexion, nous porterons attention aux façons dont l'ironie et l'humour permettent une mise à distance de l'Allemagne et du danger qu'elle représente, en tant qu'ennemi de guerre. Notre travail représente une première plongée dans l'œuvre, car aucune étude détaillée n'a été faite sur le roman de Jéhin. Les informations disponibles tant sur l'œuvre que sur le journal dans lequel le roman a été publié sont lacunaires. Il va sans dire que, dans cette optique, un travail descriptif s'avère nécessaire, afin de mettre en relief les différents éléments du roman contribuant à la constitution de l'image de l'Autre.

\section{Les personnages allemands dans le roman}

Dans son étude sur les œuvres québécoises qui portent sur la Première Guerre mondiale, Micheline Cambron affirme que «[1]a Grande Guerre est l'occasion de satire et de caricature» (Cambron 2012: 21) dans Les aventures extraordinaires de deux Canayens. Cette satire se matérialise par des «allusions à l'actualité » (Cambron 2012 : 21) déformées par la fiction. Dans cet article, nous explorerons de manière plus approfondie la dimension satirique de l'œuvre soulignée par Cambron, sans qu'elle l'ait examinée en détail.

Les personnages allemands qui ont réellement existé figurent tous dans le même chapitre du roman qui a un titre allemand : "Gott mit Huns " (AEDC: 55-60). Dans ce chapitre, l'Empereur Guillaume II s'entretient avec son fils, le Kronprinz, et avec son chancelier. Le ton humoristique est donné dès le titre du chapitre qui est un jeu de mots. Gott mit uns, qui signifie Dieu avec nous, fut la devise militaire allemande utilisée depuis le temps des chevaliers teutons jusqu'aux années 1960. Jéhin transforme le uns de la devise en Huns. La devise devient: Dieu avec les Huns. À l'époque, les Allemands étaient fréquemment comparés au peuple des Huns, considérés par les Occidentaux comme étant un peuple conquérant, sauvage et barbare. Le stéréotype de l'Allemagne militaire barbare est ici activé grâce à ce jeu de mots. Le lecteur sait, avant même 
d'amorcer sa lecture du chapitre, que l'Allemagne sera un objet de dérision dans le texte qui suit.

Dans le chapitre qui nous intéresse, les trois personnages allemands discutent de retraites stratégiques faites par l'armée allemande en France, du combat à Verdun, des espions allemands qui tentent de déterminer qui dirige l'Empire de l'espace et de la volonté de Guillaume II de dénicher un diplomate d'expérience qui pourrait négocier avec l'Empereur de l'espace qui, on l'a vu, est un simple Canadien français. La conversation des personnages allemands s'articule autour de l'histoire personnelle fictionnelle de personnes connues et de faits de guerre réels. Par exemple, le Kronprinz accuse son père de ne pas avoir respecté sa promesse de neutralité à la Belgique, ce qui est un fait réel. Toutefois, cette accusation de la part du Kronprinz relève de la fiction. D'ailleurs, dans le chapitre, les faits de guerre décrits sont tous réels, mais la manière dont les personnages allemands en discutent est fictionnelle et teintée d'ironie. Le chancelier affirme notamment: «des milliers de nos valeureux soldats se sont sacrifiés volontairement, préférant tomber sous les balles des mitrailleuses ennemies que d'être fusillés par les machines ou mitrailleuses que leurs officiers plaçaient derrière eux, ceci afin de leur donner du courage! » et Guillaume II lui répond: «Les braves gens!» $(A E D C$ : 55). Les mots «volontairement» et «braves» mettent en relief l'ironie de l'auteur, puisque le contraire de ce que veut entendre Jéhin est dit. Le lecteur comprend bien que les soldats allemands sont ici forcés d'aller au combat sous la menace des fusils de leur propre pays. À l'image de cet exemple, le chapitre en entier joue entre la réalité et la fiction et est teinté d'ironie. D'ailleurs, Jéhin n'a pas besoin de faire de longues descriptions des personnages allemands construits dans le récit et des faits dont ils discutent, car les lecteurs les connaissent déjà grâce à l'actualité; la dérision des personnages s'appuie sur des personnes et des faits connus. Ainsi, les personnages allemands sont simplement nommés par leur nom ou par leur titre officiel dans la narration, 
sans plus. On retrouve le même procédé pour les grandes batailles opposant l'armée allemande à l'armée alliée.

Le premier personnage allemand qui a réellement existé et qui joue un rôle clé dans le chapitre "Gott mit Huns» (AEDC : 55-60) est Guillaume II, aussi appelé «sa Majesté Impériale et Royale Guillaume von Hohenzollern, roi de Prusse et empereur d'Allemagne » $(A E D C: 55)$. Le nom et le titre véritables de Guillaume II, ayant régné sur l'Allemagne jusqu'en 1918, sont utilisés. Dans le récit, Guillaume II est associé par filiation à des personnages historiques connus comme Napoléon, le premier empereur des Français dans les années 1800, et Attila, le roi des Huns dans les années 400. En effet, Guillaume II prend une pose napoléonienne pendant sa discussion avec son fils et son chancelier : «il arpenta ainsi la pièce à plusieurs reprises une main derrière le dos, l'autre entre les boutons de son gilet, affectant ainsi une pose napoléonienne. (Ah! ce Bonaparte qui était tout à la fois son idole et son cauchemar!)»(AEDC : 57). Napoléon est considéré comme un grand conquérant, mais également comme un dirigeant mené par des ambitions sanglantes. Napoléon passa d'ailleurs la fin de ses jours en exil. Guillaume II est ainsi associé dans le récit, par sa pose napoléonienne, à un conquérant exceptionnel, mais aussi à un homme agressif qui perdit la guerre. L'Empereur allemand est somme toute ridiculisé, puisqu'il prend la pose d'un conquérant déchu. Par ailleurs, Guillaume II est dénommé le «successeur d'Attila» (AEDC: 58), ce qui renforce la représentation d'une Allemagne impériale conquérante, mais surtout barbare et perdante. Les soldats allemands sont d'ailleurs traités dans le chapitre de «hordes barbares » (AEDC:59), ce qui nourrit l'idée que Guillaume II serait à la tête d'un pays et d'une armée primitifs. Ainsi, Guillaume II serait le descendant d'Attila et l'armée allemande représenterait les Huns, comme l'annonçait le titre du chapitre. Le stéréotype de l'Allemagne barbare et primitive est déjà fortement ancré dans la société à la fin de la Grande Guerre et aucune explication n'est nécessaire de la part de Jéhin 
pour que le lecteur comprenne le référent. Jéhin utilise donc des modèles historico-mythiques, appelés des mythologismes (Angenot 1982: 262), pour caractériser Guillaume II, ce qui permet d'obtenir l'adhésion affective du lecteur en associant l'homme politique allemand à des personnages historiques connus (cf. Léger-Bélanger 2019).

Le deuxième personnage allemand important dans le chapitre «Gott mit uns », qui a aussi réellement existé, est le fils de Guillaume II. Le personnage qui porte le même nom que son père est toutefois seulement nommé dans le récit par son titre officiel «Kronprinz» $(A E D C: 55)$ qui désigne le prince héritier de l'Empire allemand. Dans le chapitre, le Kronprinz discute avec son père et le chancelier du front à Verdun, une position stratégique militaire que le vrai prince héritier allemand occupa à l'époque. Pendant cette discussion politique et militaire importante, le prince héritier est allongé sur une chaise longue, il fume et il boit. Il feuillette aussi un magazine de théâtre et de cinéma, et admire des portraits d'actrices. Le prince héritier est ridiculisé dans la narration par son comportement très voire trop décontracté pour le type de discussion politique qu'il a avec son père et le chancelier. L'auteur sous-entend, par sa description du comportement du prince héritier, que l'avenir de l'Allemagne n'est pas entre de bonnes mains: le Kronprinz serait une personne irresponsable et peu sérieuse. Ipso facto, le manque d'étiquette du prince héritier et son comportement nonchalant transforment une discussion militaire sérieuse en situation humoristique. En bref, c'est grâce à l'ironie que l'auteur déconstruit la menace que représente l'Allemagne - incarnée dans ses dirigeants - sur les Alliés.

Trois autres personnages d'hommes politiques et militaires allemands qui ont aussi existé dans la réalité apparaissent également dans le roman, mais de manière accessoire. Leur représentation est moins élaborée et caricaturale que celles de Guillaume II et de son fils; le rôle des trois personnages est similaire 
dans la fiction et dans la réalité. Dans le récit, Guillaume II affirme, pendant la discussion avec son chancelier, que von Bissing aurait été parfait, s'il avait été encore vivant, pour mettre en place une mission diplomatique contre le vaisseau inconnu qui leur cause tant de soucis (AEDC: 57). Dans la réalité, Moritz Ferdinand von Bissing fut un baron et un général pendant la Première Guerre mondiale, jusqu'à son décès en 1917. Dans le roman, Guillaume II choisit alors un autre homme politique allemand pour négocier avec les dirigeants de l'État de l'air, von Bernstoff (AEDC : 60). Ce second personnage, dont le nom complet est Johann Heinrich von Bernstoff, fut dans la réalité un diplomate et un ambassadeur allemand aux États-Unis jusqu'en 1917. D'ailleurs, l'ancien rôle joué en Allemagne par l'homme politique est directement mentionné dans le récit, « ex-ambassadeur» (AEDC : 60); le temps du récit et le temps réel concordent. Le troisième personnage, Paul von Hindenburg, fut dans la réalité un militaire et un homme d'État allemand au service de Guillaume II. Dans le roman, le chancelier parle à Guillaume II de la retraite des troupes allemandes ordonnée par le général Hindenburg dans le nord de la France (AEDC : 55). L'événement est décrit de manière caricaturale : les soldats allemands «ont en certaines circonstances [...] abandonné aux ennemis un certain nombre de fusils et canons, ceci pour ne pas avoir l'encombrement de les porter». La raison de l'abandon des armes par les soldats allemands est manifestement fausse ici; l'auteur se moque des stratégies de guerre allemandes. Les lecteurs de l'époque connaissent bien von Bissing, von Bernstoff et Hindenburg, puisque les trois hommes politiques et militaires allemands apparaissent alors fréquemment dans les nouvelles des journaux québécois qui traitent de l'actualité internationale. Bref, l'auteur tire son inspiration de la réalité grâce à des personnages réels, mais il ne s'y accroche pas totalement, car il évoque des actions fictives de la part de ces personnages et il use d'ironie. Ainsi, le réel est réinvesti et déconstruit dans la narration tout en étant en partie fidèle à lui-même. 


\section{Les illustrations qui représentent l'Allemagne dans le roman}

Les aventures extraordinaires de deux Canayens comportent vingt-six dessins comiques qui sont intégrés au texte dont deux images qui dépeignent de manière caricaturale l'Allemagne et qui contribuent au jeu entre réalité et fiction (les autres images ne représentent pas l'Allemagne). Les deux dessins véhiculent des représentations négatives de l'Allemagne. Leur rôle est de faire rire à propos de sujets sérieux et de rendre le récit visuellement attrayant.

La première image qui représente l'Allemagne, tirée du chapitre «Gott mit Huns », met en scène Guillaume II. Le dessin n'a pas de cadre de fond; l'accent est uniquement mis sur l'homme. Le personnage, découragé, tient une bouteille de vin étiquetée «Paris». Le militarisme allemand se détecte visuellement par l'habit militaire et par le casque à pointe prussien que porte Guillaume II. Ce dernier est assis et recourbé, au lieu d'adopter une position militaire officielle, debout et droit. Le personnage représente visuellement une Allemagne prussienne et militaire découragée, voire vaincue. À l'image du récit, la caricature s'inscrit dans le réel, puisqu'elle s'appuie sur la vie politique de l'époque, Guillaume II étant à la tête de l'Allemagne jusqu'à la fin de la Grande Guerre. Cependant, le dessin humoristique met en relief un personnage en position de faiblesse et abattu, ce qui ne correspond pas nécessairement à la réalité. La caricature rend le chef allemand moins menaçant que l'ennemi qu'il incarne dans la réalité.

La seconde image qui mérite notre attention se trouve dans un autre chapitre que celui sur lequel nous avons jusqu'à présent centré notre attention. Cette image a pour légende : «La kultur teutonne » $(A E D C: 70)$ et représente un poing dans un gant de fer qui écrase une église. Devant celle-ci, une femme, son enfant et son bébé fuient. Le mot «kultur» présente de manière négative le peuple allemand. En effet, ce mot qui désigne les valeurs du peuple allemand 
s'oppose à celui de «culture» qui traduirait des valeurs plus universelles. À l'époque, la kultur allemande était définie par les personnes non-allemandes occidentales comme étant barbare et non universelle, car elle se serait débarrassée de toute influence latine et européenne pour devenir purement germanique (Chalifoux 1993; Temkin 2015; Guerpin 2017). Le mot «kultur» que l'on trouve dans la légende est donc chargé de sens. Le motif de la barbarie, intégré dans le récit par la comparaison à Attila et aux Huns, est aussi repris dans l'image: l'Allemagne écrase tout sur son passage; elle est brutale et sans merci. Ainsi, le stéréotype persiste. Le motif de la barbarie est intégré à la fiction, mais il s'inscrit aussi dans la réalité, car il met en relief une Allemagne brutale qui est un ennemi de guerre.

\section{Le roman-feuilleton dans le journal Le Canard}

Le chapitre «Gott mit Huns» est doublement articulé : il fait partie du roman en entier, mais il est aussi inséré dans Le Canard, l'hebdomadaire dans lequel il est publié (Le Canard 11 août 1918 : 13). Le roman-feuilleton de Jéhin, qui se trouve dans une publication d'actualité, dialogue avec celle-ci; il y a un jeu de «frontières entre la fiction et la non-fiction» (Dion 2018 : 195). Les caricatures qui critiquent le régime allemand dans Le Canard révèlent d'ailleurs une certaine liberté de presse à Montréal.

Le 11 août 1918 - date à laquelle est publié le chapitre « Gott mit Huns » qui comporte la caricature de Guillaume II -, sept références directes à l'Allemagne sont faites dans le journal hors du roman-feuilleton, sans compter les nombreuses mentions de Foch et de la Grande Guerre. Parmi ces sept références, l'Allemagne est représentée dans deux caricatures, en page trois et sept du journal. La première caricature, sans titre, est guerrière et violente. Elle représente un soldat allemand souriant, identifiable grâce à la forme de son casque, qui traîne une femme par les cheveux et qui tient un enfant empalé sur une perche. L'image 
violente devient drôle par l'exagération des traits du soldat et de ses actions. La caricature met en relief une Allemagne militaire, primitive et barbare. Cette image parut pour la première fois dans Le Canard du 14 mars 1915, dans une série de deux caricatures ayant pour titre «La civilisation allemande en marche » (Le Canard 14 mars 1915 : 5). Ces dernières sont placées en diptyques : à gauche, l'image met en scène des soldats qui attaquent et dévêtent une femme et, à droite, l'image met en scène le soldat qui nous concerne. Cette fois, une déclaration supposée du général allemand von Bissing - personnage que l'on trouve dans le roman-feuilleton - est reproduite sous la caricature : «Il ne faut pas que personne se laisse aller à une sentimentalité déplacée...». Encore une fois, le contraire de ce qu'il faut comprendre est dit; l'ironie est centrale. La reproduction de la caricature dans le journal du 11 août 1918, trois ans après sa première publication, nous permet de tirer deux conclusions. Dans un premier temps, Le Canard publie des caricatures qui représentent l'Allemagne ennemie ou Guillaume II tant en fin de guerre que pendant la première année de celle-ci : l'Allemagne est un objet de dérision pendant toute la Grande Guerre et non seulement lorsque les Alliés sont en voie de remporter le conflit. Dans un deuxième temps, les motifs utilisés pour représenter l'Allemagne ennemie ne semblent pas beaucoup évoluer au fil de la guerre, puisque la même image qui représente une Allemagne barbare (le soldat brutal) est reprise dans le même journal à trois années d'intervalle. L'humour sert à faire oublier les désastres de la guerre et à pointer du doigt les coupables, Guillaume II et son armée.

La seconde caricature publiée dans le numéro du 11 août 1918 est celle d'une famille allemande de douze personnes attablées qui regardent avec appétit un minuscule oiseau placé dans une assiette au centre de la table. Seul le lecteur au fait de l'actualité peut comprendre que le dessin illustre la famine qui sévit alors en Allemagne. Ce dessin se trouve sous le bandeau : "Actualités de la semaine » et occupe la majorité de la page sept du journal. Cette image nous 
rappelle le fait que l'Allemagne envahit l'ensemble de l'espace du journal, tant celui fictif que réel, tant celui des mots que de l'image.

Les caricatures dans les pages d'actualité du journal représentent une Allemagne militaire et civile stéréotypée. La caricature de Guillaume II joue le même rôle, en stéréotypant l'Allemagne militaire qu'incarne son chef. Lorsqu'on observe les trois images qui représentent l'Allemagne et qui sont publiées dans Le Canard du 11 août 1918 - la caricature de Guillaume II dans le feuilleton, la caricature du soldat allemand et la caricature de la famille allemande -, on remarque que la caricature qui illustre une personne réelle, le chef allemand, se trouve dans la partie du journal consacrée à la fiction tandis que les deux autres caricatures qui illustrent l'Allemagne de manière plus générale, sans aucune personne qui existe réellement, sont publiées dans la partie qui traite des événements d'actualité. À chaque fois, l'ordre social n'est pas remis en question, il est seulement critiqué par une image humoristique.

Une seconde caricature de Guillaume II paraît dans Le Canard du 6 octobre 1918 :«Trop tard, Guillaume!!!» (p. 1) - date à laquelle le roman-feuilleton Les aventures extraordinaires de deux Canayens est encore publié. L'image représente Guillaume II qui se noie dans une étendue d'eau étiquetée «déchéance ». Le personnage de la Mort, un squelette avec une faux, vole au-dessus de $l^{\prime} E m p e r e u r^{2}$. La caricature permet de rejeter, du premier regard, l'Autre que représente l'Allemagne prussienne et militaire qu'incarne Guillaume II. L'image est cette fois publiée en première page du journal et ne se trouve pas dans la section dédiée au feuilleton. La caricature d'un personnage réel «sort» ainsi du roman-feuilleton et se dissémine dans le journal, dans les pages dédiées au réel. Il

\footnotetext{
${ }^{2}$ Guillaume II est souvent associé à la mort dans les caricatures. Voir par exemple à ce sujet la Une du Canard du 14 mars 1915 qui met en scène une caricature qui représente une immense tête de mort (qui fait un jeu d'illusions en étant en fait composée de personnages militaires allemands). On y lit la légende : «Une face de Mi-Carême ou le Kaiser a mauvaise mine».
} 
y a une «intrusion du réel (sous la forme privilégiée de la personne) dans la fiction » (Dion 2018 : 15), notamment par la représentation de Guillaume II dans le roman-feuilleton, mais en même temps une intrusion de la fiction dans l'univers référentiel du journal, par la représentation de Guillaume II dans une caricature ailleurs dans le journal. Guillaume II, bien qu'il soit un homme craint, devient un objet d'humour, ce qui a pour effet de minimiser la menace militaire qu'il incarne dans la réalité.

\section{Conclusion}

Somme toute, tant Jéhin que Le Canard usent des mêmes thématiques humoristiques en ce qui a trait aux représentations de l'Autre, incarnées par Guillaume II et son armée. Dans tous les cas, l'ordre social n'est pas bouleversé, ce sont des idéologies déjà présentes qui sont plutôt renforcées et qui agissent comme facteur de cohésion sociale. À l'été et à l'automne 1918, Le Canard entretient un lien étroit avec l'actualité, en reprenant les mêmes sujets que les journaux d'information, mais de manière ironique. Un jugement à l'endroit des hommes politiques allemands et de leurs actions est explicitement posé, sans toutefois que les gestes de ceux-ci ou les faits historiques soient expliqués. Les nombreuses références humoristiques faites dans le journal supposent ainsi une très bonne connaissance préalable $\mathrm{du}$ discours d'information qui traite de l'actualité. Les quotidiens d'information montréalais (sauf La Patrie) ne sont habituellement pas publiés le dimanche. Le Canard est justement publié le dimanche et prend donc en quelque sorte le relais des journaux d'information, mais par le rire. 


\section{Bibliographie}

\section{Corpus primaire}

Jéhin, J. (1918). Les aventures extraordinaires de deux Canayens : charivari littéraire et scientifique. Montréal, Imprimerie A-P. Pigeon, 114 p.

Le Canard (1893-1958).

\section{Corpus secondaire}

Angenot, M. (1982). La parole pamphlétaire: contribution à la typologie des discours modernes. Paris, Payot.

Cambron, M. (2012). «Le discours sur la Grande Guerre : demande d'histoire ». Dossier: La guerre dans la littérature québécoise. Voix et images, vol. XXXVII, no 2, p. 15-33.

Chalifoux, J.-J. (1993). «Culture : une notion polémique? ». Service social, vol. 42, no 1, p. 11-23.

Dion, R. (2018). Des fictions sans fiction ou le partage du réel. Montréal, Presses de l’Université de Montréal.

Guerpin, M. (2017). « Le Courrier musical et le premier conflit mondial (1904-1923). Propagande, mobilisation culturelle et sortie de guerre ». Revue musicale OICRM, vol. 4, n², p. 35-57.

Léger-Bélanger, E. (2019). «Les représentations de l'Allemagne dans la littérature québécoise et la presse montréalaise de l'entre-deux-guerres ». Thèse de doctorat, Montréal, Université de Montréal, Département des littératures de langue française.

Lüsebrink, H.-J. (1996). «La perception de l'Autre: jalons pour une critique littéraire interculturelle ». Tangence, $\mathrm{n}^{\circ}$ 51, p. 51-66.

McKim, A. (1919). The Canadian Newspaper Directory [tirage de 1918 sur microfilm]. Toronto, Preston Microfilming Service. 
Temkin, M. (2015). "Culture vs. Kultur, or Clash of Civilizations: Public Intellectuals in the United States and the Great War, 1917-1918 ». The Historical Journal, vol. 58, n 1, p. 157-182. 